\title{
First identification of an IMI-1 carbapenemase-producing colistin-resistant Enterobacter cloacae in China
}

Liang Huang ${ }^{1,2+}$, Xiaohui Wang ${ }^{1,2+}$, Yu Feng ${ }^{1,2}$, Yi Xie $^{3}$, Liping Xie ${ }^{4}$ and Zhiyong Zong ${ }^{1,2^{*}}$

\begin{abstract}
Background: Carbapenem resistance among the Enterobacteriaceae is a serious healthcare challenge. b/a $a_{I M I}$ is a carbapenemase gene mediating resistance to carbapenems but has not been commonly found. A bla $\left.\right|_{|M|}$-carrying Enterobacter cloacae, which was also resistant to colistin, is reported here.

Findings: E. cloacae strain WCHECl-1060 was recovered from a blood sample of a leukemia patient, who was not previously exposed to colistin. Strain WCHECl-1060 belongs to a new sequence type, ST410, and was resistant to carbapenems and colistin but was susceptible to third-generation cephalosporins. A new allelic variant of bla $a_{|M|-1}$, which has two silent mutations compared to the original bla $\left.\right|_{\mid \mathrm{MI}-1}$ variant, was found in strain WCHECl-1060. Conjugation and transformation experiments failed to transfer bla $\left.\right|_{|M|-1}$, suggesting a likely chromosome origin.
\end{abstract}

Conclusions: To our knowledge, this is the first report of an IMI-1 carbapenemase-producing colistin-resistant E. cloacae in China. Microbiological laboratories should be aware of the unusual carbapenem-resistant but third-generation cephalosporin-susceptible profiles of these IMI-producing isolates. The trend of colistin resistance among the Enterobacteriaceae should be also monitored.

Keywords: $\beta$-Lactamases, Carbapenemases, Resistance plasmids, bla $a_{|\mathrm{M}|}$ Enterobacter cloacae

\section{Findings}

Enterobacter cloacae strain WCHECl-1060 was recovered from the blood of a 30-year-old male patient with acute lymphoblastic leukemia in our hospital on October 2014. Species identification was performed using the Vitek II automated system (bioMerieux, Lyon, France) and was also confirmed by partially sequencing the $\operatorname{gyr} B$ gene as described previously [1]. In vitro susceptibility tests were performed using the Vitek II system and minimum inhibitory concentrations (MICs) of amikacin, ceftazidime, ciprofloxacin, colistin, imipenem and tigecycline were also determined using the microdilution broth method followed recommendations of the Clinical Laboratory Standards Institute [2]. Strain WCHECl-1060

\footnotetext{
*Correspondence: zongzhiy@scu.edu.cn

${ }^{\dagger}$ Liang Huang and Xiaohui Wang contributed equally to this work

${ }^{1}$ Center of Infectious Diseases, West China Hospital (Huaxi), Sichuan

University, Guoxuexiang 37, 610041 Chengdu, China

Full list of author information is available at the end of the article
}

was susceptible to ceftazidime (MIC $1 \mu \mathrm{g} / \mathrm{mL}$ ), amikacin (MIC $4 \mu \mathrm{g} / \mathrm{mL}$ ), ciprofloxacin (MIC $8 \mu \mathrm{g} / \mathrm{mL}$ ) and tigecycline (MIC $4 \mu \mathrm{g} / \mathrm{mL}$ ) but resistant to imipenem (MIC $256 \mu \mathrm{g} / \mathrm{mL}$ ) and colistin (MIC $>256 \mu \mathrm{g} / \mathrm{mL}$ ). Strain WCHECl-1060 was also susceptible to aztreonam, ceftriaxone, cefepime, piperacillin-tazobactam, gentamicin, tobramycin, levofloxacin, trimethoprim-sulphamethoxazole and minocycline, intermediate to nitrofurantoin and cefotetan, and resistant to meropenem and ertapenem as determined by Vitek II.

Strain WCHECl-1060 was screened using PCR for $b l a_{\mathrm{IMI}}$ with in-house designed primers IMI-Fn (AGAGTTCYATTCACCCATCACA) and IMI-Rn (TCTCCAATCGACCGCATGAA) and for other acquired carbapenemase-encoding genes $b l a_{\mathrm{GES}}, b l a_{\mathrm{KPC}}$, $b l a_{\mathrm{IMP}}, b l a_{\mathrm{NDM}}, b l a_{\mathrm{OXA}-48}$ and $b l a_{\mathrm{VIM}}$ as described previously [3-6]. bla IMI $_{\text {was }}$ the only carbapenemase gene detected in strain WCHECl-1060. The complete coding sequence of $b l a_{\mathrm{IMI}}$ was further amplified with an 
additional pair of in-house designed primers, IMI-up (CTGGCACGCATAGTAACCCA) and IMI-dw (ATGCCGAAAGTGCAAGCCT). Sequencing of the amplicon revealed the presence of $b l a_{\mathrm{IMI}-1}$. Of note, the $b l a_{\mathrm{IMI}-1}$ gene identified here had two silent mutations (T201C and $\mathrm{C} 459 \mathrm{~T}$, positions assigned with respect to the ATG start codon of the $b l a_{\mathrm{IMI}-1}$ gene) compared to the original $b l a_{\mathrm{IMI}-1}$ gene (GenBank accession number U50278) found in the USA and has one silent mutation (T201C) to another bla $_{\mathrm{IMI}-1}$ variant (GenBank accession JX090311) found in Taiwan. In total, three types of bla IMI-1 have been found so far and the $b l a_{\text {IMI-1 }}$ gene identified here is a new variant whose sequence has been deposited into the GenBank under the accession number KP284436.

Strain typing was performed using the multi-locus sequence typing (MLST) scheme for E. cloacae (http:// pubmlst.org/ecloacae/). Strain WCHECl-1060 was of a new sequence type, ST410, which was assigned by the curator of the MLST database.

Conjugation experiments were performed using the azide-resistant Escherichia coli strain J53 as the recipient with potential transconjugants being selected on media containing $2 \mu \mathrm{g} / \mathrm{mL}$ imipenem and $150 \mu \mathrm{g} / \mathrm{mL}$ sodium azide. Transformation experiments were carried out using plasmid DNA prepared from WCHECl-1060 by alkaline lysis, which was electroporated into $E$. coli strain DH $5 \alpha$ with potential transformants being selected on media containing $2 \mu \mathrm{g} / \mathrm{mL}$ imipenem. Both conjugation and transformation experiments failed to transfer carbapenem resistance, suggesting that $b l a_{\text {IMI-1 }}$ was likely located on the chromosome. This result was not surprising considering that most $b l a_{\mathrm{IMI}}$ genes found so far are chromosome-borne [7-9].

Currently, there are 11 assigned variants of IMI enzymes (IMI-1 to -11, http://www.lahey.org/Studies/ other.asp\#table1). In addition, carbapenemase NCM-A shares $97 \%$ amino acid identity with IMI-1 and is therefore also a type of IMI enzyme. The distribution of IMIproducing isolates is summarized in Table 1 . Two features of the distribution of IMI-producing isolates could be noticed. First, although IMI/NCM-A type enzymes have been reported for 20 years, surprisingly, only a few IMIproducing isolates have been identified since then. In contrast, another Class A carbapenemase, KPC, has been commonly found in carbapenem-resistant Enterobacteriaceae worldwide. Second, although IMI-producing isolates are not common, they have a wide geographic distribution having been found in isolates from Asia, Europe, and North and South Americas (Table 1). Several reasons could be proposed for the scarce identification of IMI-producing isolates. IMI has only weak activity against third-generation cephalosporins [7] and, based on available data, IMI-producing Enterobacter spp. are also
Table 1 The distribution of IMI-producing isolates

\begin{tabular}{|c|c|c|c|c|}
\hline IMI type ${ }^{a}$ & Host species & Location & $\begin{array}{l}\text { Accession } \\
\text { number }\end{array}$ & References \\
\hline \multirow[t]{6}{*}{1} & E. cloacae & USA & U50278 & {$[7]$} \\
\hline & E. cloacae & Taiwan & JX090311 & \\
\hline & E. cloacae & France & & {$[8]$} \\
\hline & E. asburiae & Ireland & & {$[10]$} \\
\hline & E. cloacae & Canada & KR057494 & \\
\hline & E. cloacae & China & & This study \\
\hline \multirow[t]{3}{*}{2} & E. coli & Spain & JN412066 & {$[15]$} \\
\hline & E. asburiae & USA & DQ173429 & [16] \\
\hline & E. cloacae & China & AY780889 & [12] \\
\hline 3 & E. cloacae & Hong Kong & GU015024 & [11] \\
\hline 4 & E. cloacae & Singapore & KF958750 & \\
\hline 7 & E. cloacae & Singapore & KM103296 & \\
\hline 8 & E. cloacae & UK & KP081315 & \\
\hline \multirow[t]{6}{*}{ NCM-A } & E. cloacae & Argentina & AJ536087 & \\
\hline & E. cloacae & Canada & KR057492 & \\
\hline & E. cloacae & Canada & KR057493 & \\
\hline & E. cloacae & Canada & KR057495 & \\
\hline & E. cloacae & Canada & KR057496 & \\
\hline & E. cloacae & France & Z21956 & [9] \\
\hline
\end{tabular}

${ }^{a} b / a_{I M l-5,-6,-9,-10}$ and -11 have been assigned but no sequences nor publications are available

usually susceptible to ciprofloxacin $[10,11]$. As thirdgeneration cephalosporins and fluoroquinolones are the most common antimicrobial agents used for treating human infections, the susceptibility of IMI-producing isolates to these commonly-used agents may hinder their wide dissemination. In addition, genes encoding IMI-1 and NCM-A are largely found on the chromosome rather than carried by plasmids, which may serve as an obstacle for horizontal transfer of the genes between strains and between species. Nonetheless, the current epidemiological pattern of IMI-producing strains have not been fully understood and therefore more surveillances for IMIproducing strains is warranted. The exact origin of IMI enzymes and how it emerged in Enterobacter spp. also remain unknown.

This is the first report in China of an E. cloacae clinical isolate that carries $b l a_{\mathrm{IMI}-1}$ and is resistant to colistin. Nonetheless, three IMI-producing E. cloacae strains have previously been found in China. IMI-1 was found in Taiwan (GenBank accession number JX090311) but no details such as susceptibility are available for the host strain. IMI-2 was detected in a single strain in Zhejiang Province and IMI-3 was reported from Hong Kong but the susceptibility of both strains to colistin has not been tested $[11,12]$.

The resistance to colistin of strain WCHECl-1060 is unexpected and worrying as colistin is not available for 
clinical treatment in China, the patient had no exposure to this agent and Enterobacter spp. is not intrinsically resistant to colistin [13]. Unfortunately, there is no national data on the susceptibility of Enterobacter spp. to colistin in China. As colistin is expected to become available in China soon, monitoring resistance to colistin among the Enterobacteriaceae is required at both national and local levels. Resistance to colistin imposes a great challenge for treating carbapenem-resistant Enterobacteriaceae. However, the mechanism conferring resistance to colistin among Enterobacter spp. remains largely uninvestigated although a recent study revealed resistance to colistin in an E. cloacae strain recovered in the USA is associated with cross-resistance to the host antimicrobial lysozyme [14]. Further investigation for the resistance mechanism of strain WCHECl-1060 is warranted.

In conclusion, this is the first report in China of an IMI-producing colistin-resistant E. cloacae clinical isolate. The unusual resistance profile, i.e. being resistant to carbapenems while susceptible to third-generation cephalosporins, of the Enterobacteriaceae should prompt laboratories to be aware of the presence of IMI enzymes. As colistin is crucial last resort antimicrobial against infections caused by Gram-negative bacilli, monitoring resistance to colistin among Enterobacter spp. and studies on the colistin-resistance mechanism are much needed to combat antimicrobial resistance.

\section{Availability of supporting data}

The sequence of the $b l a_{\text {IMI-1 }}$ variant in this study has been deposited into the GenBank under the accession number KP284436. The MLST profile of strain WCHECl-1060 is available at the E. cloacae MLST database (http:// pubmlst.org/ecloacae/).

\section{Abbreviations}

MICs: minimum inhibitory concentrations; MLST: multi-locus sequence typing.

\section{Authors' contributions}

$\mathrm{LH}, \mathrm{XW}, \mathrm{YF}$ and $Y X$ carried out the study and helped to draft the manuscript. PX participated in the design of the study and helped to draft the manuscript. ZZ conceived of the study, analyzed the data and drafted the manuscript. All authors read and approved the final manuscript.

\section{Author details}

${ }^{1}$ Center of Infectious Diseases, West China Hospital (Huaxi), Sichuan University, Guoxuexiang 37, 610041 Chengdu, China. ${ }^{2}$ Division of Infectious Diseases, State Key Laboratory of Biotherapy, Chengdu, China. ${ }^{3}$ Laboratory of Clinical Microbiology, Department of Laboratory Medicine, West China Hospital, Sichuan University, Chengdu, China. ${ }^{4}$ Department of Haematology, West China Hospital, Sichuan University, Chengdu, China.

\section{Acknowledgements}

This work was supported by a grant from the National Natural Science Foundation of China (Project no. 81222025), the New Century Excellent Talents Program, Ministry of Education, China (Project no. NCET-13-0399) and a grant from Sichuan Bureau of Science, China (Project no. 2013JQ0042).

\section{Competing interests}

The authors declare that they have no competing interests.

Received: 20 July 2015 Accepted: 8 November 2015

Published online: 25 November 2015

\section{References}

1. Yamamoto S, Harayama S. PCR amplification and direct sequencing of gyrB genes with universal primers and their application to the detection and taxonomic analysis of Pseudomonas putida strains. Appl Environ Microbiol. 1995;61:1104-9.

2. CLSI. Performance standards for antimicrobial susceptibility testing: twenty-third informational supplement, M100-S23. Wayne: Clinical and Laboratory Standards Institute; 2013.

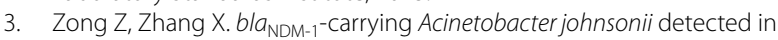
hospital sewage. J Antimicrob Chemother. 2013;68:1007-10.

4. Mendes RE, Kiyota KA, Monteiro J, Castanheira M, Andrade SS, Gales $A C$, et al. Rapid detection and identification of metallo- $\beta$-lactamaseencoding genes by multiplex real-time PCR assay and melt curve analysis. J Clin Microbiol. 2007:45:544-7.

5. Poirel L, Le Thomas I, Naas T, Karim A, Nordmann P. Biochemical sequence analyses of GES-1, a novel class A extended-spectrum $\beta$-lactamase, and the class 1 integron In52 from Klebsiella pneumoniae. Antimicrob Agents Chemother. 2000;44:622-32.

6. Bradford PA, Bratu S, Urban C, Visalli M, Mariano N, Landman D, et al. Emergence of carbapenem-resistant Klebsiella species possessing the class A carbapenem-hydrolyzing KPC-2 and inhibitor-resistant TEM-30 B-lactamases in New York City. Clin Infect Dis. 2004;39:55-60.

7. Rasmussen BA, Bush K, Keeney D, Yang Y, Hare R, O'Gara C, et al. Characterization of IMI-1 $\beta$-lactamase, a class A carbapenem-hydrolyzing enzyme from Enterobacter cloacae. Antimicrob Agents Chemother. 1996:40:2080-6.

8. Naas T, Cattoen C, Bernusset S, Cuzon G, Nordmann P. First identification of bla $a_{\mathrm{MI}-1}$ in an Enterobacter cloacae clinical isolate from France. Antimicrob Agents Chemother. 2012;56:1664-5.

9. Naas T, Nordmann P. Analysis of a carbapenem-hydrolyzing class A B-lactamase from Enterobacter cloacae and of its LysR-type regulatory protein. Proc Natl Acad Sci USA. 1994;91:7693-7.

10. Boo TW, O'Connell N, Power L, O'Connor M, King J, McGrath E, et al. First report of IMI-1-producing colistin-resistant Enterobacter clinical isolate in Ireland. Euro Surveill. 2013;18(31):20548-50.

11. Chu YW, Tung VW, Cheung TK, Chu MY, Cheng N, Lai C, et al. Carbapenemases in enterobacteria, Hong Kong, China, 2009. Emerg Infect Dis. 2011;17:130-2.

12. Yu YS, Du XX, Zhou ZH, Chen YG, Li L. First isolation of bla $a_{|M|-2}$ in an Enterobacter cloacae clinical isolate from China. Antimicrob Agents Chemother. 2006;50:1610-1.

13. Landman D, Georgescu C, Martin DA, Quale J. Polymyxins revisited. Clin Microbiol Rev. 2008;21:449-65.

14. Napier BA, Band V, Burd EM, Weiss DS. Colistin heteroresistance in Enterobacter cloacae is associated with cross-resistance to the host antimicrobial lysozyme. Antimicrob Agents Chemother. 2014;58:5594-7.

15. Rojo-Bezares B, Martin C, Lopez M, Torres C, Saenz Y. First detection of bla $a_{|M|-2}$ gene in a clinical Escherichia coli strain. Antimicrob Agents Chemother. 2012;56:1146-7.

16. Aubron C, Poirel L, Ash RJ, Nordmann P. Carbapenemase-producing Enterobacteriaceae, U.S. rivers. Emerg Infect Dis. 2005;11:260-4. 